\title{
MAIN CHARACTERISTICS AND BIOLOGICAL EFFECTS OF MARTIX METALLOPROTEINASES IN THE NERVOUS SYSTEM
}

\author{
Vanya Goranova \\ Department of Anatomy, Histology and Embryology, Medical University of Varna, \\ Bulgaria
}

\begin{abstract}
Martix metalloproteinases (MMPs), one of the subgroups of the metzincins, are a large family of zinc-dependent endoproteases with multiple roles in extracellular matrix remodelling and modulation of signalling pathways. They are able to cleave all protein components of the extracellular matrix, as well as to activate or inactivate various signaling molecules, such as receptors, growth factors and adhesion molecules. MMPs are associated with many physiological functions such as embryonic development, angiogenesis and wound healing. Therefore, these proteinases are considered to be crucial mediators in many biological processes. Elevated MMP levels have also been implicated in an increasing number of injuries and disorders, such as inflammation, cancer and auto-immune diseases. Recent investigations highlight the beneficial and detrimental effects of MMPs in the nervous system in normal and pathological conditions. This review focuses on the role of MMPs as modulators of fundamental functions in the developing and adult nervous system and their potential to improve repair or regeneration after injury.
\end{abstract}

Keywords: MMP domain structure, family members, substrates, neural plasticity

\section{INTRODUCTION}

The family of matrix metalloproteinases (MMPs), also called matrixins, has classically been described in the context of extracellular matrix (ECM) remodelling, which occurs throughout life in diverse processes ranging from tissue morphogenesis to wound healing. It has been more than 50 years since Gross and Lapiere (1) discovered collagenolytic activity during tadpole tail metamorphosis, which was later on revealed as MMP-1, the founding mem-

\footnotetext{
Address for correspondence:

Vanya Goranova Stefovska

Dept. of Anatomy, Histology and Embryology,

Medical University of Varna

55 Marin Drinov Str.

9002, Varna, Bulgaria

e-mail:vanya.goranova@mu-varna.bg
}

Received: February 11, 2015

Accepted: March 11, 2015 ber of the family of the matrixins. MMPs have been found in vertebrates, invertebrates, and plants. Till now 28 members have been discovered out of which 23 are found in human. They are distinguished from other endopeptidases by their dependence on metal ions as cofactors, their ability to ECM, and their specific evolutionary DNA sequence (2). The various MMPs exist as secreted or membrane-bound enzymes that require conversion from zymogen to active forms through proteolytic processing. The members of the MMP family are proteases (3) that collectively can degrade all constituents of the ECM. As a result of their potent proteolytic activity, abnormal MMP functions can also lead to pathological conditions. MMPs are involved in various normal and pathological processes such as morphogenesis, cell proliferation, migration, differentiation, tissue repair, angiogenesis, inflammation, tumor invasion, and apoptosis by degrading all kinds of extracellular matrix proteins, cleavage of cell surface receptors, and processing a large number of bioactive molecules 
(4-7). MMPs may affect cell migration by changing the cells from an adhesive to nonadhesive phenotype and by degrading the ECM $(8,9)$. MMPs may alter ECM microenvironment leading to cell proliferation, apoptosis, or morphogenesis (10). They may modulate the activity of biologically active molecules such as growth factors or growth factor receptors by cleaving them or releasing them from the ECM and may alter the protease activity by cleaving the enzymes or their inhibitors $(11,12)$.

MMPs are involved in a delicate balance between proteolytic and anti-proteolytic activity to confine them in time and space. When this balance is disturbed, various disease phenotypes are observed ranging from tissue destruction in chronic inflammatory conditions, such as rheumatoid arthritis and chronic obstructive pulmonary disease, to cancer metastasis and neurological disorders (13). Many different stimuli, such as growth factors, cytokines and reactive oxygen species, can trigger MMP expression in physiological as well as pathological conditions $(14,15)$. This review describes the structure of MMPs and their physiological roles in the developing and adult nervous system.

\section{STRUCTURAL FEATURES OF MMPS}

\subsection{General organization}

MMPs are largely excreted proteins with several conserved domains (Fig. 1). Structurally, MMPs are divided into three domains: an amino-terminal propeptide region, an amino-terminal catalytic domain (which contains the zinc-binding motif) and a carboxy-terminal domain, which has a high level of similarity to members of the hemopexin family and is involved in ECM substrate binding for many MMPs. All MMPs contain the catalytic domain, which is shielded off in the inactive form of the enzyme by the prodomain (13). The zinc binding motif HEXXHXXGXXH in the catalytic domain, and the "cysteine switch" motif PRCGXPD in the propeptide are common structural signatures, where three histidines in the zinc binding motif coordinate and the cysteine in the propetide coordinate with the catalytic zinc ion. This Cys- $\mathrm{Zn}^{2+}$ coordination keeps proMMPs inactive by preventing a water molecule essential for catalysis from binding to the zinc atom (15). The carboxy-terminal hemopexin-like domain is a four-bladed $\beta$-propeller structure that is present in all MMPs except MMP-7 and MMP-26 (matrilysin and matrilysin-2). A hinge region, which is short in collagenases and long in other MMPs, connects the carboxy- and amino-terminal domains. A short signal sequence ("pre-domain") lies at the amino-terminal end of the protein, before the propeptide region, and is clipped off as newly synthesized MMPs travel to the cell surface.

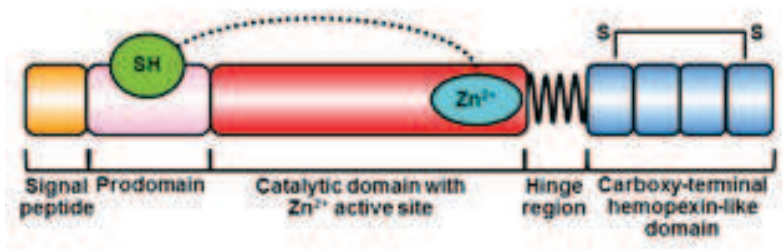

Fig. 1. Basic domain organization of MMPs.

The generic structure shown here describes most MMP family members. This includes the following main domains: signal peptide, prodomain (propeptide region) with a cysteine residue (SH), catalytic domain with $\mathrm{Zn}^{2+}$ active site, hinge region and carboxy-terminal hemopexin-like domain which contains four repeat units. The first and fourth are connected by a disulphide bridge ( $S$ $S)$. The "SH" at the propeptide region denotes the cysteine residue that ligates the zinc in the catalytic domain to keep the enzyme inactive. See Table 1 for domain arrangement of each $M M P$.

MMPs are divided into eight subgroups on the basis of their domain organization (Table 1). They contain signal peptide in the predomain, prodomain and catalytic domain which are also shared by others besides hemopexin, transmembrane and cytoplasmic domain (16). Gelatinases (MMP-2/-9) have a unique fibronectin type II-like domain inserted into the catalytic site, whereas MT-MMPs have a transmembrane domain at the carboxy terminus. Matrilysin (MMP-7) lacks the hinge and the carboxyl terminus. Uunlike other MMP groups, the membranetype (MT)-MMPs are membrane proteins. MT4MMP (MMP-17) and MT6-MMP (MMP-25) are glycosyl-phosphatidylinositol (GPI)-anchored MMPs, whereas the other MT-MMPs are transmembrane proteins (17).

\subsection{Specific domain features by groups}

Based on domain organization and substrate preference, MMP family members are grouped into 
Table 1. Domain structural classification of MMPs

MMP subgroup
Basic/Common hemopexin
domain-containing
3) Minimal-domain
4) Furin-activated
5) Vitronectin-like motif
7) Membrane Type I
8) Membrane Type II

1) Basic/Common organization: signal peptide, prodomain, catalytic domain with $\mathrm{Zn}^{2+}$ active site, hinge region and carboxy-terminal hemopexin-like module, $\mathrm{SH}=$ thiol group in the prodomain bound to $\mathrm{Zn}$ ion in the inactive state of $M M P$; 2) No hemopexin domain; 3) Gelatin-binding, Fibronectin repeat (Fn); 4) Furin-activated, Furin cleavage site (Fr); 5) Vitronectin-like (Vn) motif in the prodomain; 6) Transmembrane Type I (TM), Cytosolic (C) region; 7) Transmembrane Type I with Glycosylphosphatidylinositol (GPI) anchor; 8) Transmembrane Type II with Cysteine array (Cys) and Ig-like domain.

collagenases, gelatinases, stromelysins, matrilysins, membrane-type MMPs and "other MMPs" $(2,18)$. However, there is much overlap in substrate specificity between subgroups.

* Collagenases (MMP-1, MMP-8, MMP-13 and MMP-18 in Xenopus) cleave interstitial collagens I, II and III into characteristic $3 / 4$ and $1 / 4$ fragments but they can digest other ECM molecules and soluble proteins (19). Two other matrixins, i.e., MMP-2 and MMP-14 (MT1-MMP), have collagenolytic activity, but they are classified into other subgroups because of their domain compositions.

* Gelatinases (MMP-2 and MMP-9) readily digest gelatin (denatured collagen) with the help of their three fibronectin type II repeats that binds to gelatin/collagen. They also digest a number of ECM molecules including type IV, V and XI collagens, laminin, aggrecan core protein, etc.
MMP-2, but not MMP-9, digests collagens I, II and III in a similar manner to the collagenases. The collagenolytic activity of MMP-2 is much weaker than MMP-1 in solution, but because proMMP-2 is recruited to the cell surface and activated by the membrane-bound MT-MMPs, it may express reasonable collagenolytic activity on or near the cell surface.

* Stromelysins (MMP-3, MMP-10 and MMP11) have a domain arrangement similar to that of collagenases, but they do not cleave interstitial collagens. MMP-3 and MMP-10 are similar in structure and substrate specificity, but MMP-11 (stromelysin 3) is distantly related. The MMP-11 gene is located on chromosome 22, whereas MMP-3 and MMP-10 are on chromosome 11, along with MMP-1, $-7,-8,-12$, $-20,-26$ and -27. MMP-3 and MMP-10 digest a number of ECM molecules and participate 
in proMMP activation. MMP-11, on the other hand, has very weak activity toward ECM molecules, but cleaves serpins more readily. MMP11 has a furin recognition motif $\mathrm{RX}[\mathrm{R} / \mathrm{K}] \mathrm{R}$ at the C-terminal end of the propeptide and therefore it is activated intracellularly.

* Matrilysins (MMP-7 and MMP-26) lack a hemopexin domain. MMP-7 is synthesized by epithelial cells and is secreted apically. Besides ECM components it processes cell surface molecules such as pro- $\alpha$-defensin, Fas-ligand, protumor necrosis factor $\alpha$, and E-cadherin. MMP26 is expressed in normal cells such as those of the endometrium and in some carcinomas. It digests several ECM molecules, and unlike most other MMPs, it is largely stored intracellularly.

* MT-MMPs in mammals includes four type I transmembrane proteins (MMP-14, MMP15, MMP-16, and MMP-24) and two glycosylphosphatidylinositol-anchored proteins (MMP-17 and MMP-25). They all have a furin recognition sequence $\mathrm{RX}[\mathrm{R} / \mathrm{K}] \mathrm{R}$ at the $\mathrm{C}$-terminus of the propeptide. They are therefore activated intracellularly and active enzymes are likely to be expressed on the cell surface. All MT-MMPs except MT4-MMP (MMP-17) can activate proMMP-2. MT1-MMP (MMP-14) has collagenolytic activity on collagens I, II, and III.

* Other MMPs include seven MMPs (MMP-12, MMP-19, MMP-20, MMP-21, MMP-23, MMP-

27, the human orthologue of chick embryo MMP-22, and MMP-28) that are not grouped in the above categories although MMP-12, MMP-20 and MMP-27 have similar structures and chromosome location as stromelysins. Metalloelastase (MMP-12) is expressed primarily in macrophages, but is also found in hypertrophic chondrocytes and osteoclasts. It digests elastin and a number of ECM molecules. It is essential in macrophage migration. MMP19 digests many ECM molecules including the components of basement membranes. It is also called RASI (rheumatoid arthritis synovial inflammation) as it is found in the activated lymphocytes and plasma from patients with rheumatoid arthritis. It is recognised as an autoan- tigen in patients with rheumatoid arthritis and systemic lupus erythematosis. It is also widely expressed in many organs including proliferating keratinocytes in healing wounds. Enamelysin (MMP-20) is expressed in newly formed tooth enamel and digests amelogenin. MMP21 was originally found in Xenopus and more recently in mice and humans. It is expressed in various fetal and adult tissues and in basal and squamous cell carcinomas. It digests gelatin, but information about the action on ECM components is not known. MMP-23 is a unique member as it has unique C-terminal cysteinerich immunoglobulin-like domains instead of a hemopexin domain. The propeptide lacks a cysteine switch. It is proposed to be a type II membrane protein having a transmembrane domain at the N-terminal of the propeptide, but the enzyme is released from the cell as the membrane anchored propeptide is cleaved by a proprotein convertase. MMP-27 was first found in chicken embryo fibroblasts. Chicken MMP27 digests gelatin, casein and causes autolysis of the enzyme. Little information is available on the activity of the mammalian enzyme. Epily$\sin$ (MMP-28) is expressed in many tissues such as lung, placenta, heart, gastrointestinal tract and testis. The enzyme expressed in basal keratinocytes in skin is considered to function in wound repair. MMP-21, MMP-23 and MMP28 have a furin recognition sequence before the catalytic domain and therefore they are likely to be activated intracellularly and secreted as active enzymes.

\subsection{Classification of MMP family members}

The MMPs are zinc proteases which belong to metalloprotease family and are responsible for degradation of extracellular matrix proteins, activating proMMPs, processing various receptors and bioactive molecules (Table 2) (16). For a more complete list of MMP substrates see Nagasse et al. (15) and Yong et al. (17).

\subsection{Regulation of metalloproteinase activity}

The activity of metalloproteinases is tightly regulated, as these molecules are potent proteolytic enzymes that can cause widespread destruction. As for all secreted proteinases, the catalytic activity of 


\begin{tabular}{|c|c|c|}
\hline Member & Common name & Collagen type, proMMPs and other matrix substrates \\
\hline MMP-1 & Collagenase 1 & I, II, III, VII, VIII, X, proMMP-2/-9, Agg, Gel, PG \\
\hline MMP-2 & Gelatinase A & $\begin{array}{l}\text { I, III, IV, V, VII, X, XI, XIV, proMMP-1/-9/-13, Agg, EL, FN, } \\
\text { Gel, LN, PG, VN }\end{array}$ \\
\hline MMP-3 & Stromelysin 1 & $\begin{array}{l}\text { III, IV, IX, X, XI, proMMP-1/-7/-8/-9/-13, Agg, EL, FN, Gel, } \\
\text { LN, PG, VN }\end{array}$ \\
\hline MMP-7 & Matrilysin & IV, X, proMMP-1/-2/-9, Agg, Cas, EL, FN, Gel, LN, PG, VN \\
\hline MMP-8 & Collagenase 2 & I, II, III, V, VII, VIII, X, Agg, EL, FN, Gel, LN \\
\hline MMP-9 & Gelatinase B & IV, V, VII, X, XIV, Agg, EL, FN, Gel, PG, VN \\
\hline MMP-10 & Stromelysin 2 & III, IV, V, IX, X, proMMP-1/-8, Agg, EL, FN, Gel, LN, PG \\
\hline MMP-11 & Stromelysin 3 & IV, EL, FN, Gel, LN \\
\hline MMP-12 & Metalloelastase & IV, Cas, EL, FN, Gel, LN, PG, VN \\
\hline MMP-13 & Collagenase 3 & I, II, III, IV, VII, IX, X, XIV, proMMP-9, Agg, FN, Gel \\
\hline MMP-14 & MT1-MMP & I, II, III, proMMP-2/-13, Agg, EL, FN, Gel, LN, \\
\hline MMP-15 & MT2-MMP & proMMP-2, Agg, FN, Gel, LN \\
\hline MMP-16 & MT3-MMP & III, proMMP-2, Gel, FN \\
\hline MMP-17 & MT4-MMP & Fibrinogen/fibrin \\
\hline MMP-18 & Collagenase 4 & I \\
\hline MMP-19 & RASI 1 & IV, Gel, FN, LN \\
\hline MMP-20 & Enamelysin & Amelogenin \\
\hline MMP-21 & Xenopus MMP & Gel \\
\hline MMP-22 & Chick embryo MMP & Cas, Gel \\
\hline MMP-23 & Cysteine array MMP & Gel \\
\hline MMP-24 & MT5-MMP & proMMP-2, Gel \\
\hline MMP-25 & MT6-MMP & IV, Gel, FN \\
\hline MMP-26 & Matrilysin 2/endometase & IV, Gel, FN \\
\hline MMP-27 & Human MMP-22 & Gel, Cas \\
\hline MMP-28 & Epylisin & Cas \\
\hline
\end{tabular}

MMP-4, MMP-5 and MMP-6 were found to be identical to other MMPs. Therefore, these are not listed according to this nomenclature. MMP-27 is the human orthologue of chick embryo MMP-22. Agg, aggrecan; Gel, gelatin; PG, ptoteoglycan link protein; EL, elastin; FN, fibronectin; LN, laminin; VN, vitronectin; Cas, Casein; MT, membrane type; RASI 1, rheumatoid arthritis synovial inflammation 1.

MMPs is regulated at the following points: gene expression, compartmentalization, i.e., pericellular accumulation of enzyme, proenzyme (or zymogen) activation, and enzyme inactivation, as well as it is further controlled by substrate availability and affinity $(20,21)$.

* A first level of regulation is at their transcription, as most MMPs are not constitutively expressed but are transcribed after cell activation. Transcription of many MMPs is promoted by inflammatory cytokines, growth factors, chemokines, oncogenes and cell-cell or cell-matrix interactions (17).

* A second level of regulation is the post-translational modifications of MMPs. Many MMPs are expressed as inactive zymogens in which the cysteine residue at the propeptide region binds the zinc ion present at the catalytic site. Activating factors include the plasminogen-plasmin cascade, as well as other MMPs that disrupt the interaction between cysteine and zinc (the so-called "cysteine switch" mechanism) and then remove the propeptide region for full activation of the enzyme. Non-proteolytic compounds such as sulphydryl-reactive agents (4-aminophenylmercuric acetate) and denaturants (urea) can also activate zymogens. A subset of MMPs contains a cleavage site for furinlike prohormone convertases between the propeptide and catalytic domains; this subset includes the MT-MMPs, which are activated dur- 
ing secretion and appear on the cell surface in the active form.

* A third level of regulation of MMP activity is by the interaction of active MMPs with tissue inhibitors of metalloproteinases (TIMPs). Four TIMPs are now known and they cause inactivation by binding to the catalytic site of MMPs. Interestingly, TIMPs are also required for the activation of some MMPs. In this regard, a complex formed by TIMP-2 and the carboxyl terminus of proMMP-2 has been found to bind MT1-MMP (MMP-14) on the cell surface. An adjacent MT1-MMP molecule then removes the propeptide region of MMP-2. The proMMP/TIMP/MT-MMP trimolecular complex highlights another feature of MMP regulation: the active proteinase is found focally in the pericellular region, rather than being diffusely distributed.

In addition, other means also exist to localize MMP activity to the pericellular region. For example, activated MMP- 2 can bind the av $\beta 3$ integrin, whereas active MMP-9 can interact with the hyaluran receptor $\mathrm{CD} 44$ on the cell membrane.

\section{BIOLOGICAL EFFECTS OF MMPS IN NERVOUS SYSTEM ONTOGENY}

MMPs are expressed in the central nervous system (CNS) during development, pointing out to their possible importance in brain maturation. Expression levels of MMP-2, MMP-9, and TIMP-1 were strongest in the mouse neocortex during the embryonic stages supporting a role for these factors in the control of neuronal proliferation and migration. Their activity during early postnatal life suggests additional roles in events involved in neuronal survival and differentiation, as well as gliogenesis, including migration of astrocytes and oligodendrocyte precursors (22).

Furthermore, MMPs are rapidly upregulated after nearly all types of injury to the CNS indicating their possible beneficial effects in tissue repair. Although metzincins and TIMPs have been mostly studied in the context of nervous system disease and injury, the last decade has witnessed a growing interest of neuroscientists for their role in developmental plasticity and repair. The molecular and cellular events that support post-lesion repair of the mature
CNS recapitulate some of the processes set in motion during development. Strong evidence now indicates that the metzincin/TIMP system plays critical roles in these phenomena (23).

\subsection{Metalloproteinases and embryonic} neurogenesis

The mechanisms by which metalloproteinases regulate neurogenesis have not yet been fully defined. In concordance with the classic role of MMPs in modulating the motility of cells across tissue matrices, metalloproteinases might regulate the migration of precursor cells to their destinations during neural development. Neural stem cells express MMP2 and all four TIMPs, and the migration of an oligodendrocyte progenitor requires MMP activity in vitro. There is experimental evidence for the stimulating role of some MMPs as key molecular effectors on the proliferation and migration of embryonic neural stem/progenitor cells (Fig. 2) (24). One possibility is the modulation of the ECM and/or other guidance molecules. Many of the molecules that effect cell fate decisions in neurogenesis are substrates for metalloproteinases. There is substantial evidence that metalloproteinases regulate also the axonal growth. The metalloproteinase activity is localized to the growth cones of neurons, and inhibition of metalloproteinase activity reduces growth cone motility. In PC12 cells (a cell line derived from a pheochromocytoma of the rat adrenal medulla, that has an embryonic origin from the neural crest and contains a mixture of neuroblastic cells and eosinophilic cells) treated with nerve growth factor (NGF), neurite extension correlates with the localization of MMP-3 by immunohistochemistry to the growth cones. Active proteolytic activity demonstrated by in situ zymography with gelatin-FITC (fluorescein isothiocyanate) as a substrate has also been shown in cerebellar neurons and at the edge of growth cones from dorsal root ganglion neurons (17). MMP-9 controls survival of neurons by regulating laminin-integrin $\beta 1$ signaling during developmental neuronal death in the newborn rat hippocampus (25). MT5-MMP expression is closely associated with the formation of dendritic trees of Purkinje neurons. Growth factors and ECM molecules that promote neurite outgrowth, such as NGF and laminin, increase the expression of MMP2 in neurons. So, the formation of neurites and the 
activity of growth cones during development are associated with the expression of several metalloproteinases. This relationship is functionally coupled, as the reduction of metalloproteinase activity decreases neurite outgrowth and affects guidance decisions. It is known that several MMPs have important role in the histogenesis of the developing cerebellum. During the early postnatal period between PD3-PD12, the immunoreactivity of MMP-2 and MMP-9 was increased in both the external and internal granule layers of the cerebellar cortex. MMP-2 was mainly localized in the Bergmann glial fibers and the Purkinje cell layer, whereas MMP-9 was detected intensively in the Purkinje cell layer and the extracellular space of the molecular layer. These findings showed that MMP-2 and MMP-9 are involved in diverse neuronal functions including migration, process extension, and synaptic plasticity during the postnatal cerebellar morphogenesis (26).

The MMP/TIMP system has been shown to influence neural cell differentiation and survival. TIMP-2 acts in synergy with NGF to induce cell cycle arrest of neuronal precursors and promote neuronal differentiation, while MMP-9 and MMP-12 regulate oligodendrocyte maturation and myelination possibly through the modulation of insulin-like growth factor bioavailability (27). In vivo, postnatal programmed cell death of cerebellar granule cell precursors is reduced in MMP-9 KO mice (28). These data underscore the importance of MMPs in the control of neuronal survival.

Cell migration is another pivotal developmental function that involves metalloproteinase activities. Migration of cerebellar granule cell precursors correlates with changes in MMP-9 expression and external granular layer migration is delayed in MMP$9 \mathrm{KO}$ mice or after blockade of MMP-9 activity with neutralizing antibodies in cerebellar explants (28). Another gelatinase, MMP-2, regulates the motility of cultured non-stimulated astrocytes possibly via an interaction with $\beta 1$ integrins and the actin cytoskeleton (29). The migration of individual neuroblasts along the RMS (rostral migratory stream) from the SVZ (subventricular zone) to the olfactory bulb or those migrating radially within the olfactory bulb is perturbed after birth by furin inhibitors thought to inhibit the activation of MT-MMPs.
Perhaps the most critical role of metzincins in developmental plasticity is regulation of neurite outgrowth. Metalloproteinase activity is permissive for axonal extension via cleavage of inhibitory ECM proteins, e.g. chondroitin sulfate proteoglycans (CSPGs). MMPs also mobilize ECM-sequestered trophic factors such as epidermal growth factor (EGF). In addition, MMP-7 can proteolytically convert the proforms of brain-derived neurotrophic factor (BDNF) and NGF into the biological active forms and MMP-9 degrades active NGF (30). In turn, NGF and BDNF can stimulate the expression and activity of MMP-2, -9, and -14 (23). This proteolysis can either activate or inhibit signaling pathways. Altogether, these data reinforce the idea that matrixins and TIMPs may work in concert to regulate neurite extension/pathfinding during development.

\subsection{Other MMP functions in the developing} nervous system

A beneficial role for metalloproteinases in axonal guidance, myelinogenesis and synaptogenesis during development seems to be supported by several lines of evidence (Fig. 2). MMPs are implicated in the guidance of vertebrate retinal ganglion cell axons and different metalloproteinases function to regulate axon behaviour at distinct choice points: an MMP is important in guidance at the optic chiasm and the target, while either a different MMP is required for axons to make the turn in the mid-diencephalon (31). Another role for MMPs in CNS development might lie in myelinogenesis, the process whereby oligodendrocytes extend several processes from their soma that reach and enwrap axons to form myelin. The initial expansion of oligodendroglial processes is immense and could require remodelling of the brain matrix by MMPs. This hypothesis has been tested and oligodendrocytes were found to express MMP-9 during the period of myelinogenesis. Furthermore, the inhibition of MMP activity in vitro prevented the extension of oligodendroglial processes (17). In parallel with myelinogenesis, metalloproteinases also participate in axon elongation. Early studies noted the presence of proteolytic activity at neuronal growth cones during attachment and reattachment events. Some of the activity is probably contributed by metalloproteinases, as interference with MMP activity inhibited growth-cone mo- 
tility. Inducers of neuronal differentiation and axonal outgrowth, such as NGF, laminin or retinoic acid, enhanced the expression of MMP-2, MMP-3 and MMP-9 by dorsal root ganglion neurons (17). Although these data implicate metalloproteinases in the creation of penetrable paths for axonal elongation, metalloproteinases can also regulate guidance cues for growth cones. Ephrins are guidance molecules that bind to receptor tyrosine kinases of the Eph family. When the growth cone of a neuron that expresses Eph receptors encounters ephrin ligands on the surface of another cell, this facilitates the adherence of the cells to each other and bidirectional signalling to occur. The growth cone then overcomes these adhesive forces and breaks away from the ephrin surface. MMPs and TIMPs may work in concert to regulate neurite extension/pathfinding during development. During brain maturation NMDA receptors promotes dendritic spine development and remodelling through MMP-mediated intercellular adhesion molecule-5 (ICAM-5) cleavage (32).

Among known MMP substrates are several proteins that play important roles in synaptogenesis, synaptic plasticity, and long-term potentiation (LTP). MMP-directed cleavage of these proteins can impact the formation and function of synapses within the brain. Pyramidal neurons in the hippocampus, and other large neurons, are surrounded by perineuronal nets that are composed of brevican, tenascin$\mathrm{R}$, and laminin, each of which is subject to proteolytic cleavage by MMPs. Tenascin- $\mathrm{R}$ knockout mice show deficits in learning and memory and LTP, as do at least two MMP knockouts. Impaired LTP is also seen in BDNF knockout mice, which is interesting in that pro-BDNF can be processed into mature BDNF by several MMPs and thereby regulate activation of the high-affinity BDNF receptor TrkB. At the synaptic level, MMP substrates also include ephrins, Eph receptors, and cadherins, which are also involved in synapse development and plasticity (33).

MMP-2 has been suggested to be largely involved in the development of human brain microvessels. Endothelial cells and pericytes tightly interplay in both angiogenesis mechanisms, by ECM proteolysis, and angiogenesis regulation, by local (autocrine/ juxtacrine) vascular endothelial growth factor (VEGF) action (34).

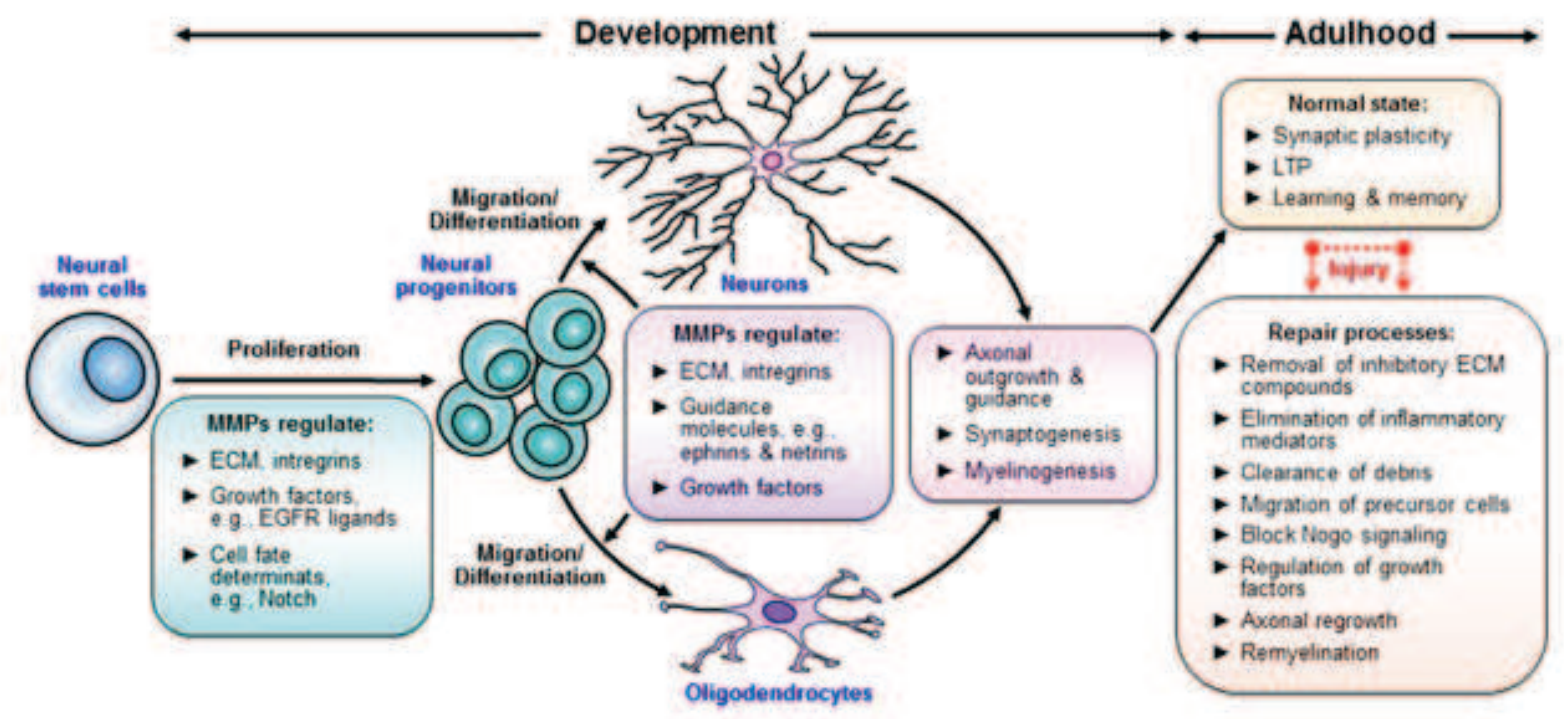

Fig. 2. Functional involvement of MMPS in the developing and adult nervous system

MMPs regulate developmental and repair processes such as the genesis of progentors from a neural stem cell, migration and differentiation of the progenitors into neurons and oligodendrocytes. They participate also in the subsequent formation of axons, synapses and myelin. The factors and mechanisms by which MMPs control these processes are indicated. Similarly, following an injury, metalloproteinases are involved in the regenerative events through specific mechanisms. ECM, extracellular matrix; EGFR, epidermal growth factor receptor; LTP, long-term potentiation; Nogo, neurite outgrowth inhibitor 


\section{ROLE OF MMPS IN THE ADULT NERVOUS SYSTEM}

\subsection{Plasticity within the normal adult brain}

MMPs/TIMPs play a significant role in LTP and learning. Several reports support a role for MMPs in physiological LTP at diverse brain regions. The pivotal role for MMP-9 in LTP was first established by Nagy et al. (35) who revealed that MMP9 protein levels and proteolytic activity were rapidly increased by stimuli that induce late-phase LTP. Furthermore, using MMP-9 KO mice, as well as broadspectrum MMP inhibitors, the authors reported deficient late-phase LTP in hippocampal slices at the CA3 to CA1 pathway. Taken together, these data indicate that MMPs are involved in various aspects of LTP, with MMP-9 needed for late-phase LTP and MMP-3 for LTP induction (36). In this context, it is noteworthy that MMP-3 acts upstream of MMP-9, i.e., MMP-3 regulates MMP-9 activation. Given the role of MMPs in hippocampal LTP, it comes as no surprise that MMP inhibition has also been linked to memory deficits in behavioral learning paradigms $(35,37)$. Sympathetic neurons secrete MMP-2 and MT1-MMP to control nerve sprouting via pro-NGF conversion (38).

There is also data that indicates a role for metalloproteinases in synaptic plasticity related to the learning and memory (Fig. 2) (39,40). Both the MMP-9 transcript and protein are upregulated in the cell bodies and dendrites of hippocampal neurons within hours of experimental kainate stimulation, and the protease appears to have a role in the dendritic remodelling (41). In traumatic brain injury involving the hippocampus, the resultant reactive synaptic plasticity corresponds to increased MMP-3 expression (42).

In addition to developmental plasticity discussed above, there are multiple physiological and pathological phenomena relying on plasticity within the adult brain, including learning and memory, epileptogenesis, and drug addiction. Several experimental paradigms, mimicking some aspects of plasticity, have also been identified, with LTP and long-term depression (LTD) being the most prominent. Myriad molecules have been proposed to play a role in brain plasticity. Most studies focus on external information conveyed to neurons predominantly by means of neurotransmitters, neurotrophins, cytokines and steroids. However, much less is known about a major extracellular molecular brain structure surrounding neuronal synapses, i.e., ECM and cell adhesion molecules. Since plasticity is associated with post-synaptic structural alterations, proteolytic ECM modifications likely contribute to plasticity.

\subsection{Plasticity in the post-injured adult brain}

In the injured adult CNS, the metalloproteinases have mainly detrimental activities but some beneficial functions are also possible (Fig. 2) (Table 3) (43, 44). What could they be? It is possible that metalloproteinases enable the migration of precursor cells to injured sites to replenish lost cells (45). Accumulating data now indicate that MMPs may play beneficial roles by mediating the neurogenic response in the subventricular zone after brain injury such as trauma, ischemia, and hemorrhage. By allowing neuroblasts to expand and migrate, MMPs should facilitate the endogenous recovery response in a damaged brain $(46,47)$.

A role in facilitating axonal regrowth, remyelination and angiogenesis is also possible on the basis of the functions of these proteases in development (48). Another possible role of MMPs in the adult CNS might involve their potential to affect signal transduction by, for example, linking G-protein-coupledreceptor signalling with the EGF pathway. In this context, the engagement of G-protein-coupled receptors has been shown to activate a membrane-associated metalloproteinase that converts proheparinbinding EGF to its mature form, triggering EGF signaling (17). Regulation of synaptic plasticity could be another function of metalloproteinases in CNS injury (49). MMPs could also participate in terminating inflammation in the CNS, in contrast to the perpetuation of inflammation. The prolonged incubation of IL-1 $\beta$ with MMP-3, and to a lesser extent MMP-2 and -9 , resulted in IL-1 $\beta$ degradation injury (50). Furthermore, MMP-2 binds to the chemokine macrophage chemoattractant protein 3 (MCP-3) and cleaves its first four amino-terminal amino acids. The cleaved MCP3 binds to several chemokine receptors, acting as an antagonist. So, MMPs expressed in the CNS might serve to abolish a chemotactic gradient for leukocyte entry. Interestingly, MMP-9 has been reported to truncate the amino terminus of IL-8, leading 
to a 30-fold potentiation of IL-8 activity. Conversely, MMP-9 degraded chemokines, such as gro- $\alpha$, leaving RANTES (also known as SCYA5) and MCP-2 intact (17).

Matrix metalloproteinases have been suggested as key facilitators of successful axonal regeneration for several reasons. First, MMPs add to the clearance of cellular and matrix debris at the site of injury. Second, all major constituents of the glial scar are substrates of at least one MMP $(51,52)$ and increased MMP activity reduces glial scarring and associated inhibitory molecules. In particular, the CSPGs have been denoted for their strong inhibitory impact on axonal regeneration (53) and are subject to cleavage from ECM heparin sulphate proteoglycans) or the conversion of inactive progrowth factors to their active forms, e.g., NGF and BDNF $(42,52,54)$. Finally, as the directed outgrowth of axons requires extensive motility and infiltration within the nervous tissue, MMPs can invoke: "focalized" proteolysis at the growth cone, thereby reorganizing the ECM to facilitate attachment and motility of the growing axon (55). Experimental evidence demonstrates that MMP-2 promote adult axon regeneration by olfactory ensheathing cells (56).

Although thus far only described in the brain and spinal cord, it can be assumed that these MMP actions can be extrapolated to the entire CNS, in-

Table 3. Metalloproteinases in the nervous system

\begin{tabular}{|ll}
\hline Beneficial effects & \multicolumn{1}{c}{ Detrimental effects } \\
$*$ Cell-fate specification & $*$ Inflammation \\
$*$ Migration of neuronal precursors & $*$ Disruption of blood-brain barrier \\
$*$ Angiogenesis & $*$ Neuronal death \\
$*$ Survival of cells & $*$ Oxidative stress \\
$*$ Axonal growth & $*$ Toxicity \\
$*$ Myelinogenesis & $*$ Stroke \\
$*$ Signaling & $*$ Trauma \\
$*$ Learning and memory & $*$ Tumorigenesis \\
$*$ Terminate inflammation & $*$ Neurodegenerative diseases
\end{tabular}

MMPs play crucial beneficial roles in CNS development, normal functioning and regeneration but they are detrimental mediators in CNS inflammation, infection and injury destroying neurons, axons and myelin and resulting in various disease conditions.

by MMPs. Indeed, the repulsion of neurite growth cones and "masking" of neurite-promoting laminin by CSPGs, can be (partially) abrogated by MMP (52). Third, MMPs are also able to degrade myelin-derived inhibitory ligands released by degenerating CNS axons, such as neurite outgrowth inhibitor (Nogo), myelin-associated glycoprotein, oligodendrocyte myelin glycoprotein (OMgp), and myelin basic protein (MBP) $(42,52,53)$. Fourth, besides disarming inhibitory ECM and signaling molecules, MMPs can also unmask, activate, or release others that have a beneficial effect on CNS repair. As such, they can indirectly provide neurotrophic support to regenerating axons via the release of sequestered growth factors (e.g., release of basic fibroblast growth factor (bFGF) cluding the retina and optic nerve. Indeed, stimulation of axonal regeneration after optic nerve transection in rats, by intravitreal transplantation of a peripheral nerve segment, coincides with significantly enhanced MMP activity in the regenerating optic nerve as compared with the nonregenerating optic nerve (53). More specifically, MMP-2, MMP-9, and to a lesser extent also MMP-1 and -3, are upregulated in the proximal optic nerve stump and at the site of the optic nerve injury, while TIMP-1 and -2 are suppressed. This immediate local upregulation of MMP2 and MMP-9 by reactive astrocytes, that is coinciding with post injury inflammation, is thought to clear tissue debris, in concert with invading macrophages. The later burst in gelatinase activity (i.e., 8 
days post injury), which colocalizes with ECM compounds of the glial scar, might serve to break down and dissolve the glial scar, thereby clearing the path for regenerating axons (53). Remarkably, gelatinase activity only rarely colocalizes with regenerating optic nerve axons, in contrast to regenerating peripheral nerves, where growth cones secrete MMP-2 and MMP-3 to facilitate the progression of dorsal root ganglia cell axons on the peripheral nerve basal lamina (57). Instead, reactive astrocytes at the site of injury appear to be the major source of gelatinases in the CNS (53). Taken together, MMPs can potentially play part in many different aspects of successful axonal regeneration. In the optic nerve, it is already evident that they can add to the conversion of the nerve environment from a repressive to a facilitative substratum for axon growth. Moreover, endogenous MMP2 , produced by retinal astrocytes, seems to be essential to neurite outgrowth in an in vitro mouse retinal explant model (58).

Metzincins also play a role in post-injury plasticity, particularly via the regulation of the glial scar. The glial scar effectively confines the lesion, but also constitutes a major obstacle for axon regeneration, partially due to the accumulation of inhibitory molecules, e.g., CSPGs, myelin ligands. MMP-9 contributes to the early deleterious effects of spinal cord injury and to astrocyte motility during glial scar formation (59). Accordingly, MMP-2 KO mice exhibit reduced spontaneous axon regeneration and functional recovery with a concomitant increase in CSPG levels after moderate spinal cord injury.

A successful regeneration after neuronal injury concomitant with axonal degeneration, implies the generation of new synapses in order to regain neuronal functionality. As synaptogenesis depends on dynamical changes of the ECM and cleavage of pericellular protein-like growth factors and adhesion molecules, a role for MMP-2 in this elaborate process has been proposed, but only limited evidence has been gathered. Additionally to de novo synaptogenesis of newly regenerated axons, reorganization of synaptic contacts from neighboring axons also occurs, defined as post-injury synaptic plasticity, a process in which MMP-9 has recently been implied. Indeed, enhanced synaptic activity and subsequent tissue remodelling, triggered by kainate administration, results in an upregulation of MMP-9 mRNA, protein and activity in the adult rat hippocampal dentate gyrus at the site of granule cell dendrites, implicating a role for MMP-9 in activity-dependent remodelling of dendritic architecture with possible effects on synaptic physiology (41).

Remyelination is an important process during nerve repair and regeneration in the nervous system and has been associated with several MMPs $(60,61)$. Since migration and maturation of oligodendrocyte precursors and the ensheatment of axons by their processes form an essential part of the remyelinating event and need extensive ECM remodeling, proteases, and MMPs in particular, have been suggested as promising candidate molecules herein (42). MMP9 facilitates remyelination in part by processing the inhibitory neuron-glial antigen 2 proteoglycan (62) and activates cell signaling promoting migration of Schwann cells after injury (63).

In summary, metalloproteinases in the CNS have clear beneficial functions during development and might also have positive effects after injury. These effects contrast with the better-described detrimental roles of metalloproteinases in the CNS. Altogether, these findings highlight positive roles for matrixins in cell-mediated injury repair through the mobilization of endogenous neural cell precursors and the remodelling of the lesion environment by grafted cells.

\section{CONCLUSIONS AND FUTURE PERSPECTIVES}

Matrixins and their inhibitors are both foes and friends in nervous system physiopathology. However, many questions on the biology of these enzymes remain open and addressing them represents a tremendous challenge for future research. We still have very limited knowledge of their in vivo substrates and an incomplete understanding of the spatio-temporal pattern of expression of most MMPs. Many questions regarding the mechanisms of metalloproteinase action in health and disease need to be answered. What signal transduction pathways are activated upon intracellular and extracellular metalloproteinase proteolytic processing? What are the other functions of non-active proteinases and what proteins do they interact with at the plasma membrane? Do matrixins elicit activities independent of proteolysis in the nervous system? Increasing our basic knowledge 
Main characteristics and biological effects of martix metalloproteinases in the nervous system

on the biology of MMPs should improve the efficacy of potential therapeutic strategies for promoting the beneficial or inhibiting the detrimental effects of metalloproteinase activity in the nervous system.

\section{REFERENCES}

1. Gross J, Lapiere C. Collagenolytic activity in amphibian tissues: a tissue culture assay. Proc Natl Acad Sci USA. 1962;48 (6):1014-22.

2. Sekhon BS. Matrix metalloproteinases - an overview. Res Rep Biol. 2010;1:1-20.

3. Rawlings ND, Barrett AJ, Bateman A. MEROPS: the database of proteolytic enzymes, their substrates and inhibitors. Nucleic Acids Res. 2012;40:D343-50.

4. Brinckerhoff CE, Matrisian LM. Matrix metalloproteinases: a tail of a frog that became a prince. Nat Rev Mol Cell Biol. 2002;3(3):207-14.

5. Stamenkovic I. Extracellular matrix remodelling: the role of matrix metalloproteinases. J Pathol. 2003;200:448-64.

6. Malemud CJ. Matrix metalloproteinases (MMPs) in health and disease: an overview. Front Biosci. 2006;11:1696-1701.

7. Van Hinsbergh VW, Koolwijk P. Endothelial sprouting and angiogenesis: matrix metalloproteinases in the lead. Cardiovasc Res. 2008;78(2):203-12.

8. Tomlinson ML, Garcia-Morales C, Abu-Elmagd M, Wheeler GN. Three matrix metalloproteinases are required in vivo for macrophage migration during embryonic development. Mech Dev. 2008;125:1059-70.

9. Rodríguez D, Morrison CJ, Overall CM. Matrix metalloproteinases: what do they not do? New substrates and biological roles identified by murine models and proteomics. Biochim Biophys Acta. 2010;1803(1):39-54.

10. Mannello F. Multipotent mesenchymal stromal cell recruitment, migration, and differentiation: what have matrix metalloproteinases got to do with it? Stem Cells. 2006;24(8):1904-7.

11. $\mathrm{Vu} \mathrm{TH}, \mathrm{Werb} Z$. Matrix metalloproteinases: effectors of development and normal physiology. Genes Dev. 2000;14:2123-33.

12. Page-McCaw A, Ewald AJ, Werb Z. Matrix metalloproteinases and the regulation of tissue remodelling. Nat Rev Mol Cell Biol. 2007;8(3):221-33.
13. Klein T, Bischoff R. Physiology and pathophysiology of matrix metalloproteases. Amino Acids. 2011;41:271-90.

14. Sternlicht MD, Werb Z. How matrix metalloproteinases regulate cell behavior. Annu Rev Cell Dev Biol. 2001;17:463-516.

15. Nagase H, Visse R, Murphy G. Structure and function of matrix metalloproteinases and TIMPs. Cardiovasc Res. 2006;69:562-73.

16. Kumud P, Adlakha N, Mittal A. Multi class classification approcach for classification of ADAMs, MMPs and their subclasses. IACSIT. 2010;2(3):302-7.

17. Yong VW, Power C, Forsyth P, Edwards DR. Metalloproteinases in biology and pathology of the nervous system. Nat Rev Neurosci. 2001;2(7):502-11.

18. Verma RP, Hansch C. Matrix metalloproteinases (MMPs): chemical-biological functions and (Q) SARs. Bioorg Med Chem. 2007;15(6):2223-68.

19. Visse R, Nagase H. Matrix metalloproteinases and tissue inhibitors of metalloproteinases: structure, function, and biochemistry. Circ Res. 2003;92(8):827-39.

20. Chakraborti S, Mandal M, Das S, Mandal A, Chakraborti T. Regulation of matrix metalloproteinases: an overview. Mol Cell Biochem. 2003;253(1-2):269-85.

21. Ra HJ, Parks WC. Control of matrix metalloproteinase catalytic activity. Matrix Biol. 2007;26(8):587-96.

22. Bednarek N, Clement $Y$, Lelievre V, Olivier P, Loron G, Garnotel R et al. Ontogeny of MMPs and TIMPs in the murine neocortex. Ped Res. 2009;65:296-300.

23. Rivera S, Khrestchatisky M, Kaczmarek L, Rosenberg GA, Jaworski DM. Metzincin proteases and their inhibitors: foes or friends in nervous system physiology? J Neurosci. 2010;30:15337-57.

24. Ingraham CA, Park GC, Makarenkova HP, Crossin KL. Matrix metalloproteinase (MMP)-9 induced by Wnt signaling increases the proliferation and $\mathrm{mi}-$ gration of embryonic neural stem cells at low $\mathrm{O}_{2}$ levels. J Biol Chem. 2011;286:17649-57.

25. Murase S, McKay RD. Matrix metalloproteinase- 9 regulates survival of neurons in newborn hippocampus. J Biol Chem. 2012;287:12184-94.

26. Ayoub AE, Cai TQ, Kaplan RA, Luo J. Developmental expression of matrix metalloproteinas- 
es 2 and 9 and their potential role in the histogenesis of the cerebellar cortex. J Comp Neurol. 2005;481(4):403-15.

27. Larsen PH, DaSilva AG, Conant K, Yong VW. Myelin formation during development of the CNS is delayed in matrix metalloproteinase- 9 and -12 null mice. J Neurosci. 2006;26:2207-14.

28. Vaillant C, Meissirel C, Mutin M, Belin MF, Lund LR, Thomasset N. MMP-9 deficiency affects axonal outgrowth, migration, and apoptosis in the developing cerebellum. Mol Cell Neurosci. 2003;24(2):395-408.

29. Ogier C, Bernard A, Cholle, AMT, Hanessian LED, Charton S, Khrestchatisky G et al. Matrix metalloproteinase-2 (MMP-2) regulates astrocyte motility in connection with the actin cytoskeleton and integrins. Glia. 2006;54:272-84.

30. Bruno MA, Cuello AC. Activity-dependent release of precursor nerve growth factor, conversion to mature nerve growth factor, and its degradation by a protease cascade. Proc Natl Acad Sci USA. 2006;103(17):6735-40.

31. Hehr CL, Hocking JC, McFarlane S. Matrix metalloproteinases are required for retinal ganglion cell axon guidance at select decision points. Development. 2005;132:3371-9.

32. Tian L, Stefanidakis $M$, Ning L, Van Lint $P, N y-$ man-Huttunen $\mathrm{H}$, Libert $\mathrm{C}$ et al. Activation of NMDA receptors promotes dendritic spine development through MMP-mediated ICAM-5 cleavage. J Cell Biol. 2007;178:687-700.

33. Ethell IM, Ethell DW. Matrix metalloproteinases in brain development and remodeling: synaptic functions and targets. J Neurosci Res. 2007;85:2813-23.

34. Girolamo F, Virgintino D, Errede M, Capobianco C, Bernardini N, Bertossi $\mathrm{M}$ et al. Involvement of metalloprotease- 2 in the development of human brain microvessels. Histochem Cell Biol. 2004;122:261-70.

35. Nagy V, Bozdagi O, Matynia A, Balcerzyk M, Okulski P, Dzwonek J et al. Matrix metalloproteinase-9 is required for hippocampal late-phase long-term potentiation and memory. J Neurosci. 2006;26(7):1923-34.

36. Conant K, Wang Y, Szklarczyk A, Dudak A, Mattson MP, Lim ST. Matrix metalloproteinase-dependent shedding of intercellular adhesion molecule- 5 occurs with long-term potentiation. Neuroscience. 2010;166:508-21.
37. Bozdagi O, Nagy V, Kwei KT, Huntley GW. In vivo roles for matrix metalloproteinase- 9 in mature hippocampal synaptic physiology and plasticity. J Neurophysiol. 2007;98:334-44.

38. Saygili E, Schauerte P, Pekassa M, Rackauskas G, Schwinger RH, Weis J et al. Sympathetic neurons express and secrete MMP-2 and MT1-MMP to control nerve sprouting via pro-NGF conversion. Cell Mol Neurobiol. 2011;31:17-25.

39. Meighan SE, Meighan PC, Choudhury P, Davis CJ, Olson ML, Zornes PA et al. Effects of extracellular matrix-degrading proteases matrix metalloproteinases 3 and 9 on spatial learning and synaptic plasticity. J Neurochem. 2006;96(5):1227-41.

40. Wang XB, Bozdagi O, Nikitczuk JS, Zhai ZW, Zhou Q, Huntley GW. Extracellular proteolysis by matrix metalloproteinase-9 drives dendritic spine enlargement and long-term potentiation coordinately. Proc Natl Acad Sci USA. 2008;105(49):19520-5.

41. Szklarczyk A, Lapinska J, Rylski M, McKay RD, Kaczmarek L. Matrix metalloproteinase-9 undergoes expression and activation during dendritic remodeling in adult hippocampus. J Neurosci. 2002;22(3):920-30.

42. Yong VW. Metalloproteinases: mediators of pathology and regeneration in the CNS. Nat Rev Neurosci. 2005;6(12):931-44.

43. Agrawal SM, Lau L, Yong VW. MMPs in the central nervous system: where the good guys go bad. Semin Cell Dev Biol. 2008;19(1):42-51.

44. Zhang $\mathrm{H}$, Adwanikar $\mathrm{H}$, Werb Z, Noble-Haeusslein LJ. Matrix metalloproteinases and neurotrauma: evolving roles in injury and reparative processes. Neuroscientist. 2010;16(2):156-70.

45. Barkho BZ, Munoz AE, Li X, Li L, Cunningham LA, Zhao X. Endogenous matrix metalloproteinase (MMP)-3 and MMP-9 promote the differentiation and migration of adult neural progenitor cells in response to chemokines. Stem Cells. 2008;26(12):3139-49.

46. Lee SR, Kim HY, Rogowska J, Zhao BQ, Bhide $\mathrm{P}$, Parent JM et al. Involvement of matrix metalloproteinase in neuroblast cell migration from the subventricular zone after stroke. J Neurosci. 2006;26(13):3491-5.

47. Wang L, Zhang ZG, Zhang RL, Gregg SR, Hozeska-Solgot A, LeTourneau Y et al. Matrix metalloproteinase 2 (MMP2) and MMP9 secreted by erythropoietin-activated endothelial cells pro- 
mote neural progenitor cell migration. J Neurosci. 2006;26:5996-6003.

48. Cunningham LA, Wetzel M, Rosenberg GA. Multiple roles for MMPs and TIMPs in cerebral ischemia. Glia. 2005:50(4):329-39.

49. Vaillant C, Didier-Bazes M, Hutter A, Belin MF, Thomasset N. Spatiotemporal expression patterns of metalloproteinases and their inhibitors in the postnatal developing rat cerebellum. J Neurosci. 1999;19:4994-5004.

50. Ito A, Mukaiyama A, Itoh $Y$, Nagase H, Thogersen IB, Enghild JJ et al. Degradation of interleukin 1beta by matrix metalloproteinases. J Biol Chem. 1996;271(25):14657-60.

51. Duchossoy Y, Horvat JC, Stettler O. MMP-related gelatinase activity is strongly induced in scar tissue of injured adult spinal cord and forms pathways for ingrowing neurites. Mol Cell Neurosci. 2001;17:945-56.

52. Pizzi MA, Crowe MJ. Matrix metalloproteinases and proteoglycans in axonal regeneration. Exp Neurol. 2007;204(2):496-511.

53. Ahmed Z, Dent RG, Leadbeater WE, Smith C, Berry $M$, Logan A. Matrix metalloproteases: degradation of the inhibitory environment of the transected optic nerve and the scar by regenerating axons.

Mol Cell Neurosci. 2005;28:64-78.

54. Shubayev VI, Myers RR. Matrix metalloproteinase-9 promotes nerve growth factor-induced neurite elongation but not new sprout formation in vitro. J Neurosci Res. 2004;77:229-39.

55. Murphy G, Gavrilovic J. Proteolysis and cell migration: creating a path? Curr Opin Cell Biol. 1999;11:614-21.

56. Pastrana E, Moreno-Flores MT, Gurzov EN, Avila J, Wandosell F, Diaz-Nido J. Genes associated with adult axon regeneration promoted by olfactory ensheathing cells: a new role for matrix metalloproteinase 2. J Neurosci. 2006;26:5347-59.

57. De Groef L, Van Hove I, Dekeyster E, Stalmans I, Moons L. MMPs in the neuroretina and optic nerve: modulators of glaucoma pathogenesis and repair? Invest Ophthalmol Vis Sci. 2014;55(3):1953-64.

58. Gaublomme D, Buyens T, De Groef L, Stakenborg M, Janssens E, Ingvarsen S et al. Matrix metalloproteinase 2 and membrane type 1 matrix metalloproteinase co-regulate axonal outgrowth of mouse retinal ganglion cells. J Neurochem. 2014;129(6):966-79.

59. Hsu JY, Bourguignon LY, Adams CM, Peyrollier K, Zhang H, Fandel T et al. Matrix metalloproteinase- 9 facilitates glial scar formation in the injured spinal cord. J Neurosci. 2008;28(50):13467-77.

60. Lehmann HC, Köhne A, Bernal F, Jangouk P, Meyer Zu Hörste G, Dehmel T et al. Matrix metalloproteinase- 2 is involved in myelination of dorsal root ganglia neurons. Glia. 2009;57:479-89.

61. Verslegers M, Lemmens K, Van Hove I, Moons L. Matrix metalloproteinase- 2 and -9 as promising benefactors in development, plasticity and repair of the nervous system. Prog Neurobiol. 2013;105:60-78.

62. Larsen PH, Wells JE, Stallcup WB, Opdenakker G, Yong VW. Matrix metalloproteinase-9 facilitates remyelination in part by processing the inhibitory NG2 proteoglycan. J Neurosci. 2003;23(35):11127-35.

63. Mantuano E, Inoue G, Li X, Takahashi K, Gaultie, A, Gonias SL et al. The hemopexin domain of matrix metalloproteinase- 9 activates cell signaling and promotes migration of schwann cells by binding to lowdensity lipoprotein receptor-related protein. J Neurosci. 2008;28:11571-82. 